\title{
METHOD OF CALCULATION OF MAGNETIC SYSTEMS OF VOLUME MODELS BY FINITE DIFFERENCE METHOD TAKING INTO ACCOUNT SEPARATION OF DISSIMILAR MEDIA
}

\author{
Gennady Nikitenko ${ }^{1}$, Evgeny Konoplev ${ }^{1}$, Pavel Konoplev ${ }^{1}$, Anatoly Permyakov ${ }^{2}$, \\ Alexander Lysakov ${ }^{1}$, Ekaterina Sergienko ${ }^{1}$ \\ ${ }^{1}$ Stavropol State Agrarian University, Russia; ${ }^{2}$ Yaroslav-the-Wise Novgorod State University, Russia \\ nikitenko_gv@mail.ru, konoplev82@mail.ru, s_lysakov@mail.ru
}

\begin{abstract}
The article presents a method for calculating the magnetic systems of volume models by the finite difference method, taking into account the separation of dissimilar media for an axially symmetric system. A special feature of the presented calculation method is the automation of determining the boundaries of the media of volumetric models by taking into account the magnetic resistance of adjacent nodes relative to the calculated node. The results of the program for calculating the magnetic field pattern are presented on the example of a threedimensional model of a synchronous generator with a two-core magnetic system used as part of a wind generator. Three-dimensional graphical dependences of the change in the magnetic induction value inside the stator winding coil of a synchronous generator are constructed for a rotor with an external magnetic core, for a rotor with an internal magnetic core, and for a rotor with two magnetic cores simultaneously. The calculation of the magnetic system is presented in article, the method of the example synchronous generator with dual magnetic system, determining the maximum value of magnetic induction, penetrating the winding of the synchronous generator in the direction of the $r$ axis. Based on the picture of the three-dimensional distribution of the magnetic induction vector and the constructed three-dimensional graphs of the changes of the values of the magnetic induction vector along the $\mathrm{r}$ axis for an external magnetic core, the magnetic core and the double-core magnetic system is determined by the efficiency of the use of the dual magnetic system, where the magnetic induction inside the stator winding increases in average 2 times, which entails an increase in the power of the synchronous generator 4 times.
\end{abstract}

Keywords: simulation; magnetism; calculation; generator; induction.

\section{Introduction}

Calculation of magnetic systems of electric machines, in particular synchronous generators on permanent magnets, is one of the most important tasks in the field of design. There are programs that allow to perform calculation of volumetric models of magnetic systems, such as ELCUT and the like, but their high cost and complexity in the development limit their use. The solution is to develop methods for calculating magnetic systems by the finite difference method [1-6].

The elements of the magnetic systems of synchronous generators are heterogeneous, they contain permanent magnets, a magnetic core made of electrical steel, copper windings, and air gaps. The standard model for calculating the finite difference method of magnetic systems does not allow for a reliable calculation, since it is impossible to take into account the separation of the magnetic system media and this method requires improvement. Let us consider the method of calculating the axisymmetric sector system of a synchronous generator with a two-core magnetic system. In the article [7], a planar model of a magnetic system was previously calculated using the finite difference method. In this article, we present a method for calculating the volume model of a given sector of a synchronous generator and the results of calculating the magnetic induction vector from the normal component.

The problem of calculating the magnetic potential by the finite difference method is that it is impossible to determine whether the adjacent points of the calculated node belong to a particular medium or to a section of media. The introduction of additional points to adjacent points of the calculation node in the calculation of the magnetic system by the finite difference method allows to determine the belonging of adjacent points to a particular medium, or to determine their location on the media section.

Let us consider the use of the finite difference method on the example of calculating the model of a synchronous generator on permanent magnets.

\section{Materials and methods}

A three-dimensional model of the calculated sector of a synchronous generator with a two-core magnetic system is presented, and the rotor with external and internal rings, the stator and the synchronous generator are also shown in Figure 1. 

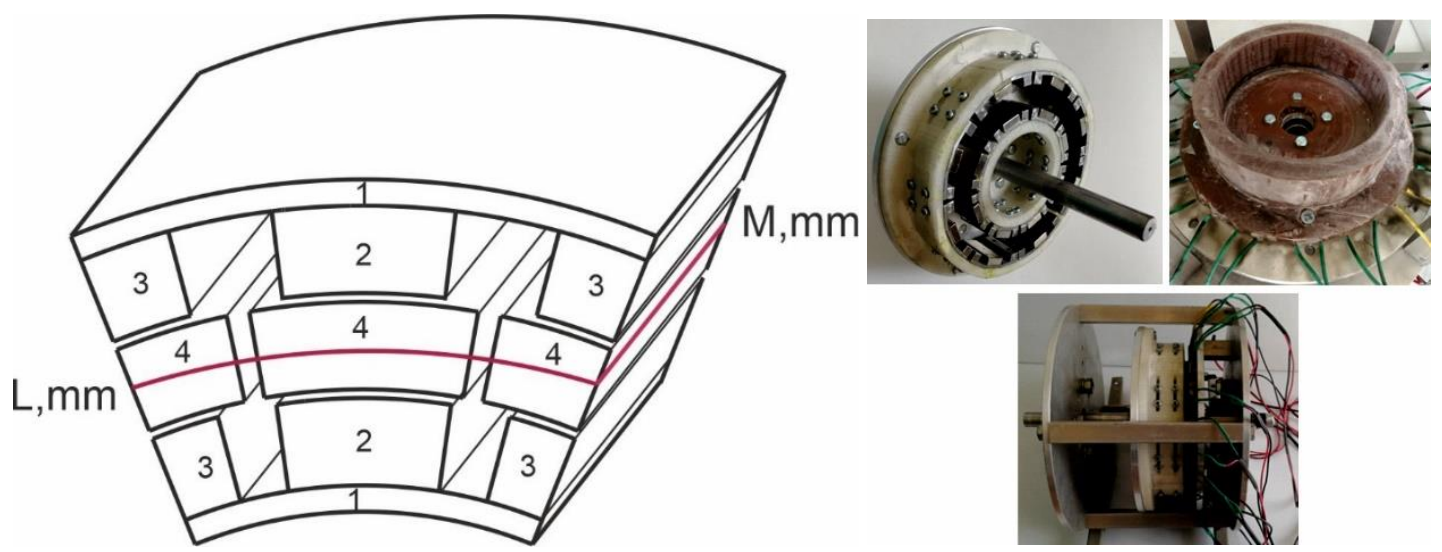

Fig.1. Volume model of the synchronous generator sector: 1 - magnetic core; 2 - permanent magnets; 3 -pseudo-poles; 4 - stator windings rotor; stator and synchronous generator assembly

The synchronous generator consists of a magnetic core 1, on which neodymium magnets 2 and additional poles 3 made of electrical steel are fixed, between the external and internal core there are windings 4 of the stator made of copper. The stator windings are stationary, the generator is operated due to the movement of the magnetic system of the rotor relative to the stator of the generator.

A model for calculating the magnetic potential in the generator nodes for bulk bodies is shown in Figure 2.

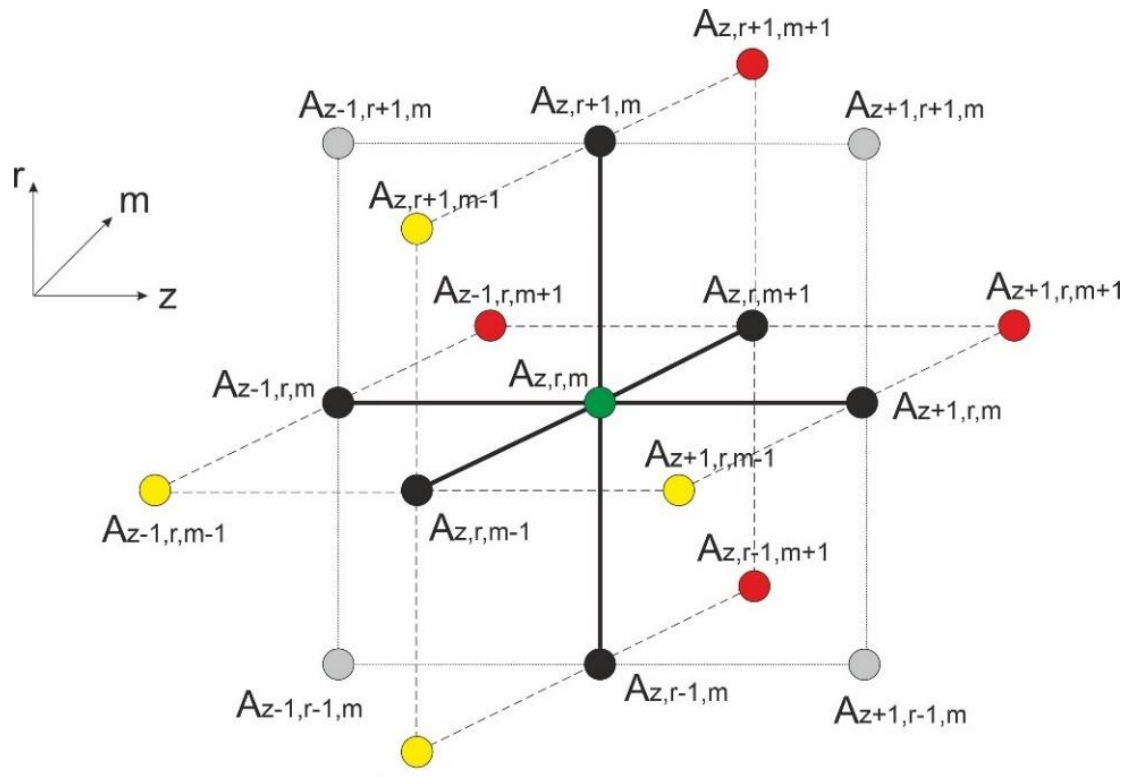

$A z,-1, m-1$

Fig. 2. Model for calculating the magnetic potential in a node for three-dimensional shapes

An improvement of the finite difference method for calculating the magnetic potential at a node for bulk bodies is the introduction of additional points $A_{z-1, r+1, m} ; A_{z, r+1, m+1} ; A_{z+1, r+1, m} ; A_{z-1, r, m+1}$; $A_{z, r+1, m-1} ; A_{z+1, r, m-1} ; A_{z, r-1, m-1} ; A_{z-1, r, m-1} ; A_{z+1, r, m+1} ; A_{z, r-1, m+1} ; A_{z+1, r-1, m+1} ; A_{z-1, r+1, m}$ allowing to determine whether adjacent nodes lie $A_{z, r+1, m} ; A_{z+1, r, m} ; A_{z, r-1, m} ; A_{z-1, r, m}$; $A_{z, r, m+1} ; A_{z, r, m-1}$ the calculation node on the section of magnetic media.

To determine whether, for example, the point $A_{z+1, r, m}$ lies on a section of media, or belongs to one medium, the magnetic resistance of the material is determined at points $A_{z+1, r-1, m} ; A_{z+1, r, m-1}$; $A_{z+1, r+1, m} ; A_{z+1, r, m+1}$. If the additional melon points of the adjacent point of the calculated node have the same magnetic resistance, then the point $A_{z+1, r, m}$ belongs to the same medium, but if the magnetic resistances are different, then this point lies on the media section, which must be taken into account when calculating the magnetic system. 
Based on the Poisson and Laplace equations, we write an expression for determining the magnitude of the magnetic potential in a node by the finite difference method, taking into account the section of heterogeneous media for bulk objects.

$$
\begin{aligned}
A_{z, r, m} & =\left(A_{z, r+1, m}\left[v_{A_{z-1, r+1, m}}+v_{A_{z, r+1, m+1}}+v_{A_{z+1, r+1, m}}+v_{A_{z, r+1, m-1}}\right]+\right. \\
+ & A_{z+1, r, m}\left[v_{A_{z+1, r+1, m}}+v_{A_{z+1, r}, m+1}+v_{A_{z+1, r-1, m}}+v_{A_{z+1, r}, m-1}\right]+ \\
+ & A_{z, r-1, m}\left[v_{A_{z, r-1, m+1}}+v_{A_{z+1, r-1, m}}+v_{A_{z, r-1, m-1}}+v_{A_{z-1, r-1, m}}\right]+ \\
+ & A_{z-1, r, m}\left[v_{A_{z-1, r+1, m}}+v_{A_{z-1, r, m+1}}+v_{A_{z-1, r-1, m}}+v_{A_{z-1, r, m-1}}\right]+ \\
+ & A_{z-1, r, m}\left[v_{A_{z-1, r+1, m}}+v_{A_{z-1, r, m+1}}+v_{A_{z-1, r-1, m}}+v_{A_{z-1, r, m-1}}\right]+ \\
+ & A_{z-1, r, m}\left[v_{A_{z-1, r+1, m}}+v_{A_{z-1, r, m+1}}+v_{A_{z-1, r-1, m}}+v_{A_{z-1, r, m-1}}\right]+ \\
+ & \left.A_{z, r, m-1}\left[v_{A_{z, r+1, m-1}}+v_{A_{z+1, r, m-1}}+v_{A_{z, r-1, m-1}}+v_{A_{z-1, r, m-1}}\right]\right) / \\
& \frac{1}{3}\left(\left[v_{A_{z-1, r+1, m}}+v_{A_{z, r+1, m+1}}+v_{A_{z+1, r+1, m}}+v_{A_{z-1, r, m+1}}+\right.\right. \\
& +v_{A_{z, r+1, m-1}}+v_{A_{z+1, r, m-1}}+v_{A_{z, r-1, m-1}+}+v_{A_{z-1, r, m-1}}+ \\
+v_{A_{z+1, r, m+1}} & \left.\left.\quad v_{A_{z, r-1, m+1}}+v_{A_{z+1, r-1, m+1}}+v_{A_{z-1, r+1, m}}\right]\right) \cdot\left(6-\frac{\Delta r+\Delta m+r \cdot \sin (\Delta z)}{3 i}\right)
\end{aligned}
$$

where $v$-magnetic resistance of the material;

$r, z, m$ - coordinates of the model;

$i$ - grid step.

Denoting

$$
\begin{aligned}
& v_{\sum v_{A_{z, r+1, m}}}=v_{A_{z-1, r+1, m}}+v_{A_{z, r+1, m+1}}+v_{A_{z+1, r+1, m}}+v_{A_{z, r+1, m-1}} ; \\
& v_{\Sigma v_{A_{z}+1, r, m}}=v_{A_{z+1, r+1, m}}+v_{A_{z+1, r, m+1}}+v_{A_{z+1, r-1, m}}+v_{A_{z+1, r, m-1}} \text {; } \\
& v_{\sum v_{A_{z, r-1, m}}}=v_{A_{z, r-1, m+1}}+v_{A_{z+1, r-1, m}}+v_{A_{z, r-1, m-1}}+v_{A_{z-1, r-1, m}} \text {; } \\
& v_{\sum v_{A_{z-1, r}, m}}=v_{A_{z-1, r+1, m}}+v_{A_{z-1, r, m+1}}+v_{A_{z-1, r-1, m}}+v_{A_{z-1, r, m-1}} \text {; } \\
& v_{\sum v_{A_{z, r}, m+1}}=v_{A_{z, r+1, m+1}}+v_{A_{z+1, r, m+1}}+v_{A_{z, r-1, m+1}}+v_{A_{z-1, r, m+1}} \text {; } \\
& v_{\sum v_{A_{z, r}, m-1}}=v_{A_{z, r+1, m-1}}+v_{A_{z+1, r, m-1}}+v_{A_{z, r-1, m-1}}+v_{A_{z-1, r, m-1}} \text {; } \\
& v_{\sum v_{A_{z, r}, m}}=v_{A_{z-1, r+1, m}}+v_{A_{z, r+1, m+1}}+v_{A_{z+1, r+1, m}}+v_{A_{z-1, r, m+1}}+ \\
& +v_{A_{z, r+1, m-1}}+v_{A_{z+1, r, m-1}}+v_{A_{z, r-1, m-1}+}+v_{A_{z-1, r, m-1}}+ \\
& +v_{A_{z+1, r, m+1}}+v_{A_{z, r-1, m+1}}+v_{A_{z+1, r-1, m+1}}+v_{A_{z-1, r+1, m}} \text {. }
\end{aligned}
$$

We get the following expression

$$
\begin{gathered}
A_{z, r, m}=\left(A_{z, r+1, m} v_{\sum v_{A_{z, r}+1, m}}+A_{z+1, r, m} v_{\sum v_{A_{z}+1, r, m}}+A_{z, r-1, m} v_{\sum v_{A_{z, r-1, m}}}+\right. \\
\left.+A_{z-1, r, m} v_{\sum v_{A_{z-1, r}, m}}+A_{z, r, m+1} v_{\sum v_{A_{z, r}, m+1}}+A_{z, r, m-1} v_{\sum v_{A_{z, r}, m-1}}\right) / \\
\left(\left(\frac{1}{3} v_{\sum v_{A_{z, r}, m}}\right)\left(6-\frac{\Delta r+\Delta m+r \cdot \sin (\Delta z)}{3 i}\right)\right)
\end{gathered}
$$

The resulting expression is used to calculate the magnetic potential of non-current-carrying parts.

Expression (3) is used to calculate the magnetic potential of current-carrying surfaces, expression (4) is used to calculate the magnetic potential of permanent magnets. 


$$
\begin{aligned}
& A_{z, r, m}=\left(A_{z, r+1, m} v_{\sum v_{A_{z, r+1, m}}}+A_{z+1, r, m} v_{\sum v_{A_{z+1, r, m}}}+A_{z, r-1, m} v_{\sum v_{A_{z, r-1, m}}}+\right. \\
& \left.+A_{z-1, r, m} v_{\sum v_{A_{z-1, r}, m}}+A_{z, r, m+1} v_{\sum v_{A_{z, r, m+1}}}+A_{z, r, m-1} v_{\sum v_{A_{z, r}, m-1}}\right) / \\
& \left(\left(\frac{1}{3} v_{\sum v_{A_{z, r}, m}}\right)\left(6-\frac{\Delta r+\Delta m+r \cdot \sin (\Delta z)}{3 i}\right)\right)-\frac{h_{r} h_{z} h_{m} J}{v_{A_{z, r}, m}}, \\
& A_{z, r, m}=\left(A_{z, r+1, m} v_{\sum v_{A_{z, r+1, m}}}+A_{z+1, r, m} v_{\sum v_{A_{z}+1, r, m}}+A_{z, r-1, m} v_{\sum v_{A_{z, r-1, m}}}\right. \\
& \left.+A_{z-1, r, m} v_{\sum v_{A_{z-1, r, m}}}+A_{z, r, m+1} v_{\sum v_{A_{z, r}, m+1}}+A_{z, r, m-1} v_{\sum v_{A_{z, r, m-1}}}\right) / \\
& \left(\left(\frac{1}{3} v_{\sum v_{A_{z, r}, m}}\right)\left(6-\frac{\Delta r+\Delta m+r \cdot \sin (\Delta z)}{3 i}\right)\right)-\frac{h_{r} h_{z} h_{m} H_{C}}{v_{A_{z, r}, m}},
\end{aligned}
$$

where $h_{r}, h_{z}, h_{m}$ - scale along the axes;

$H_{c}$ - coercive force of a permanent magnet;

$j$ - current density in the conductor.

\section{Results and discussion}

Based on the obtained expressions, translating them into a computer model using the Object Pascal programming language in Delphi 10, with the number of calculated nodes 107640, the graphical dependences of the change in the magnetic induction vector over the normal component are presented. $B_{r}$ are presented, taking into account the fact that $\mathrm{B}=\operatorname{rot} \mathrm{A}$. The plane, in which the magnetic induction vector is constructed from the normal component, is shown by the red line in Figure 3 . The $L$ value is $45 \mathrm{~mm}$, the $M$ value is $40 \mathrm{~mm}$.

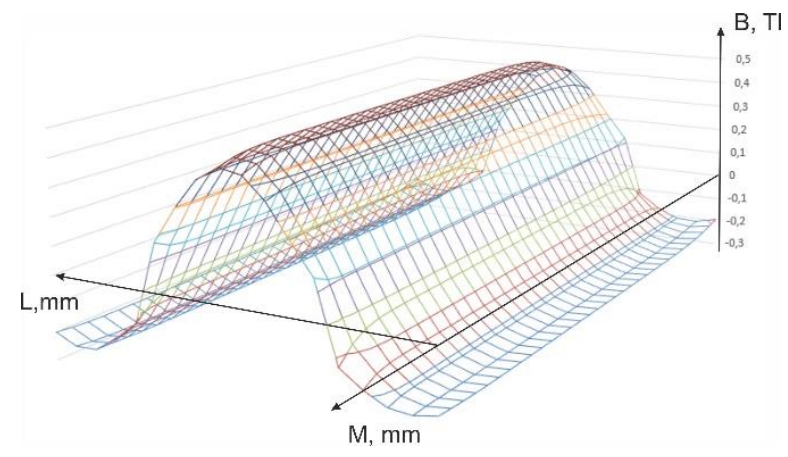

Fig. 3. Change in the magnetic induction vector $B_{r}$ along the internal cross-section of the stator winding of a synchronous generator with a double-core magnetic rotor system
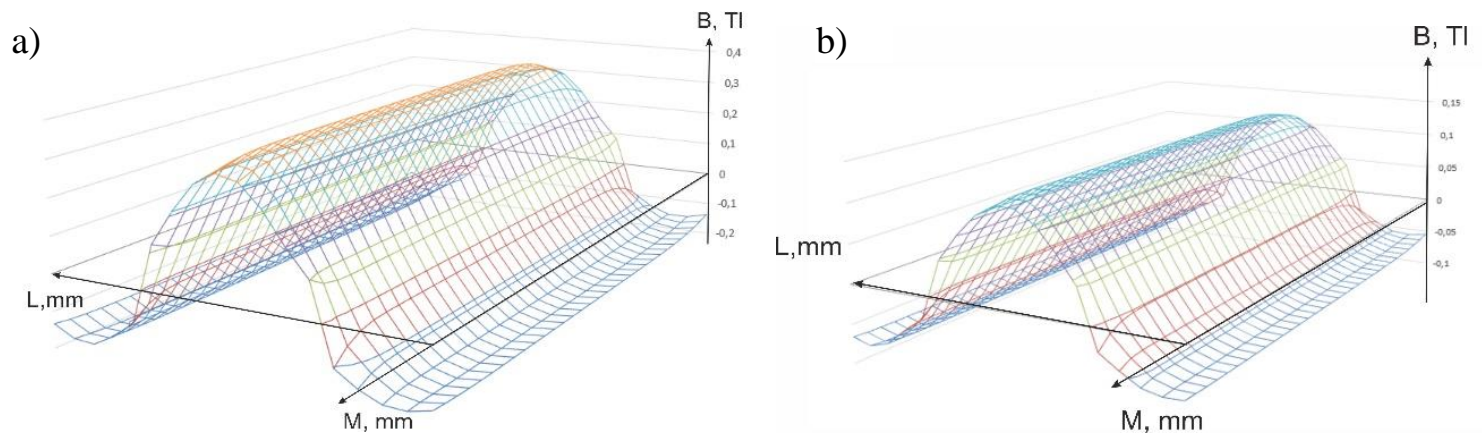

Fig. 4. Change in the magnetic induction vector $B_{r}$ along the internal cross-section of the stator winding of the synchronous generator: a - rotor with an external magnetic core;

$\mathrm{b}$ - rotor with an internal magnetic core

The normal component of the magnetic induction vector $B_{r}$ for the rotor of a synchronous generator with a two-core magnetic system, shown in Figure 3, reaches a value of $0.51 \mathrm{Tl}$, when using a rotor with an external magnetic core, this value is $0.33 \mathrm{Tl}$, when using a rotor with an internal magnetic core, the value of the magnetic induction vector was $0.15 \mathrm{Tl}$. Thus, the use of a two-core magnetic system of the rotor of a synchronous generator makes it possible to increase the value of the normal component of the 
magnetic induction vector inside the stator winding by an average of 2 times, which leads to an increase in the power of the synchronous generator.

\section{Conclusions}

1. An improved method for calculating the magnetic systems of axisymmetric volumetric models is presented, which allows taking into account the media section of the model under study.

2. On the basis of the obtained mathematical dependences a model is constructed and graphical dependences of the change in the normal component of the magnetic induction vector $B_{r}$ along the cross section of the stator winding of the synchronous generator sector are presented. The use of a two-core magnetic system allows to increase this value to $0.51 \mathrm{~T}$.

\section{References}

[1] Pavlenko I.V., Girka I.O., Trush, O.V. Exact analytical calculation and numerical modelling by finite-difference time-domain method of the transient transmission of electromagnetic waves through cold plasmas. Journal of plasma physics. T.8. 2020.

[2] Zubkov Y.V., Makarichev Y.A., Antropov V.E. Finding electromagnetic loads and magnetic-field factors in design of integrated brushless excitation DC generator. Proceedings - 2019 International Ural Conference on Electrical Power Engineering, UralCon. 2019. 8877639, pp. 217-222.

[3] Yousefian M., Zarchi H.A., Gorginpour H. Modified steady-state modelling of brushless doublyfed induction generator taking core loss components into account. IET Electric Power Applications, 2019. 13(9), pp. 1402-1412.

[4] Abdalla S.A., Abdullah S.S. Performance improvements of induction motor drive supplied by hybrid wind and storage generation system based on mine blast algorithm. Energies, 2019. 12(15),2947.

[5] Nikitenko G., Konoplev E., Salpagarov V., Lysakov A. Solar and wind stand-alone power system. Engineering for Rural Development No.18, 2019. pp. 1456-1462.

[6] Pat. 2680642 Russian Federation, IPC H02K 1/27, H02K 21/12, H02K 16/02 Synchronous generator with dual magnetic system/Nikitenko G. V., Konoplev V. E., Salpagarov K. V., Konoplev, P. V., Bobryshev, A. V., applicant and patent holder FGBOU IN Stavropol GAU. No. 2016152824, application. 30.12.16, publ. 25.01.18, Byul. No. 3.

[7] Nikitenko G., Konoplev E., Salpagarov V., Konoplev P., Bobryshev A. Method of calculating magnetic system using finite difference method. Engineering for Rural Development №19, 2020. pp. 1373-1380.

[8] Bakhvalov Y.A., Grechikhin V.V., Yufanova A.L. The Method of Fundamental Solutions using the Vector Magnetic Dipoles for Calculation of the Magnetic Fields in the Diagnostic Problems Based on Full-Scale Modelling Experiment. IOP Conference Series: Materials Science and Engineering. 127(1),012002. 2016.

[9] Aditya K., Peschiera B., Youssef M., Williamson S.S. Modelling and calculation of key design parameters for an inductive power transfer system using finite element analysis-A comprehensive discussion. 2015 IEEE Transportation Electrification Conference and Expo, ITEC 2015

[10]Zhao J., Zhang X., Zhang J., Gao W., Qu S. Field analysis of tubular permanent magnet linear synchronous motor. 2010 International Conference on Modelling, Identification and Control, ICMIC 2010. c. 290-293. 